\title{
Circular Effect Generation for Color Images
}

\author{
Gwanggil Jeon \\ Department of Embedded Systems Engineering, Incheon National University \\ 119 Academy-ro, Yeonsu-gu, Incheon 406-772, Korea \\ gjeon@incheon.ac.kr
}

\begin{abstract}
This paper presents a novel circular effect generation approach. In general, pixel location in an image can be represented with $i$ and $j$ coordinate. To manipulate $(i, j)$ coordinate system easier, we transform $(i, j)$ coordinate system into $(\rho, \theta)$ coordinate system. Two parameters are used for generating circular effect: $R$ and $T$. After applying selected $R$ and $T$ in $(\rho, \theta)$ coordinate system, $\left(\rho_{2}, \theta_{2}\right)$ are obtained. Finally, $\left(\rho_{2}, \theta_{2}\right)$ signals are inverse-transformed into $(i, j)$ coordinate system and $\left(i_{2}, j_{2}\right)$ is obtained. Experimental results introduce performance comparison.
\end{abstract}

Keywords: Image enhancements, circular effect, image effect, coordinate system, performance assessment

\section{Introduction}

Image processing techniques edit or alter original images, whether their forms are analog, digital pictures or illustrations [1-2]. In this paper we study circular effect generation methodology which changes original images into cone effect applied ones. This manipulation is one of applications of image generation techniques to original pictures in order to generate an illusion effect.

In general, image effects are normally taken with digital cameras which originally obtain images [3-8]. However, users of computers may want to give image effects. Some methods apply transparency or negative effect. By the ability of machine device such as computers, scanner, or digital cameras, one may give or remove image effects from the original image.

There are several examples of image effects application. One example is shader effect, which is normally called bloom effect. The bloom is a computer visual effect which is employed in computer game applications, and it can be used for high dynamic range (HDR) image demos to remake a color image artifact of actual cameras. It is said that the bloom effect makes feathers of light stretching from the edges of shiny regions in an original image. One of practical application is a gaming system which is able to render 3D graphics using floating point frame buffers, in order to harvest HDR images. To generate bloom effect, the HDR contents in the frame buffer are rolled with a convolution kernel in a post-processing stage, before transforming to RGB color space.

In this paper, we study circular effect generation method. The rest of the paper is organized as follows. Section 2 presents the proposed method for circular effect generation. Section 3 provides experimental results and some discussions are provided. Finally, conclusion remarks are given in Section 4.

\section{Proposed Circular Effect Generation Method}

The circular effect can be given by commercial image processing tools. To generate circular effect, it is used gradients concepts. We apply this generated effect onto exiting 
images. To this end, we use two parameters, theta $(T)$ and rotation $(R)$. Figure 1 shows the block diagram of the proposed method.

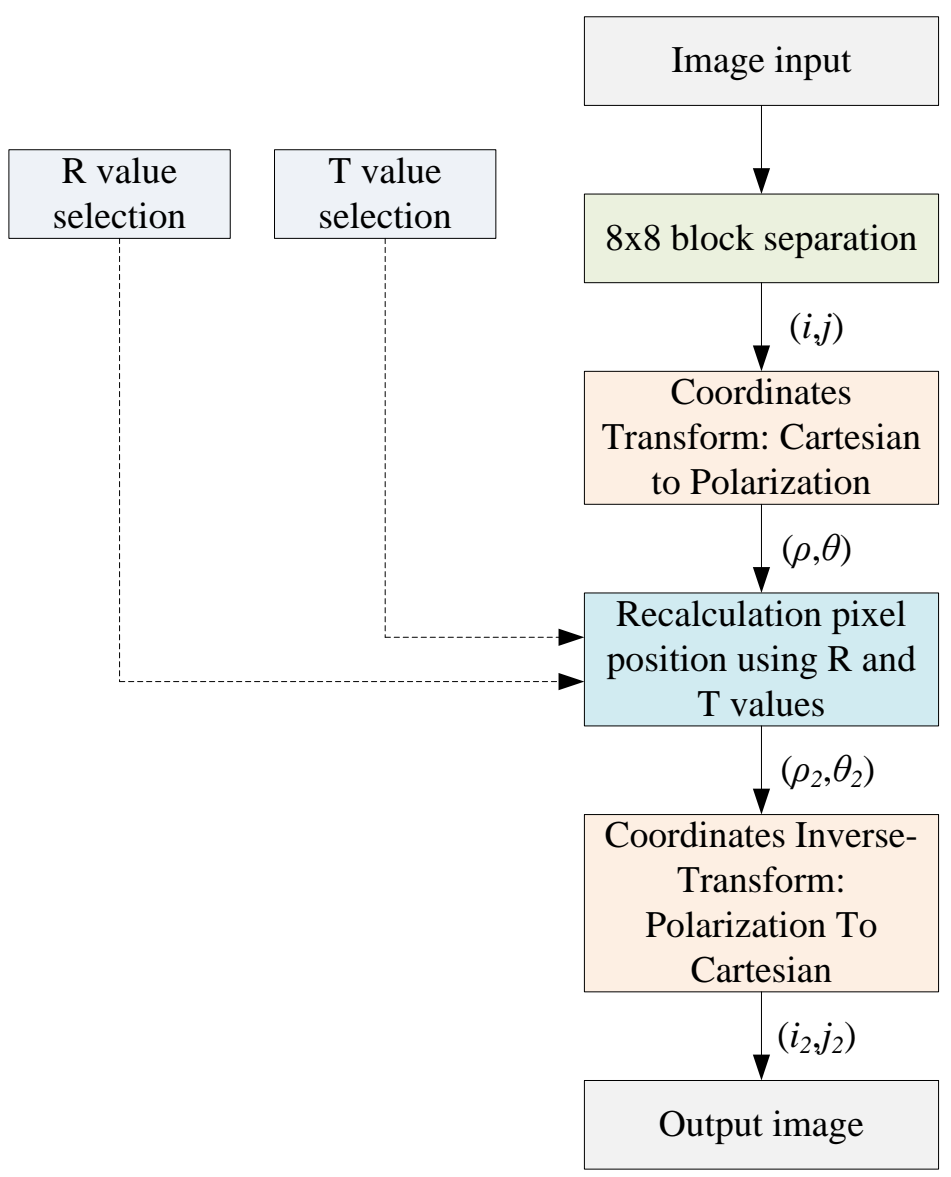

Figure 1. Block Diagram of the Proposed Method

The proposed method consists of four steps.

\section{Step1:}

After existing original image is obtained, $8 \times 8$ block separation is applied. The center pixel position of the chosen block is recalculated for giving circular effect to the existing original image.

Step2:

In this step, coordinate transform is applied. The employed coordinate transform is Cartesian coordinate to Polarization coordinate. After this process, all location components of $(i, j)$ is transformed to $(\rho, \theta)$ where $\rho$ and $\theta$ are angle and radius. The $(\rho, \theta)$ coordinate system is normally used circular, cylindrical, or spherical symmetry. For these situations it is frequently easier to utilize a diverse coordinate system. 


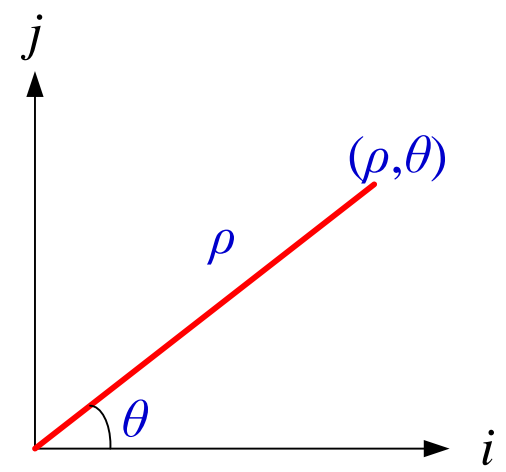

Figure 2. Example of $(i, j)$ and $(\rho, \theta)$ Coordinate Conversion

In $(\rho, \theta)$ coordinates, a point in the plane is calculated by its distance $\rho$ from the origin and the angle $\theta$ in radians between the line from the origin to the point and the $i$-axis. Figure 2 shows $(i, j)$ and $(\rho, \theta)$ coordinate system. Here, one can use traditional trigonometry to obtain conversions from $(i, j)$ and $(\rho, \theta)$ coordinate:

$$
\begin{aligned}
& \rho=\sqrt{i^{2}+j^{2}} \\
& \theta=\tan ^{-1}\left(\frac{j}{i}\right)
\end{aligned}
$$

After calculating $\left(\rho_{2}, \theta_{2}\right),\left(i_{2}, j_{2}\right)$ coordinate values are computed as follows:

$$
\begin{aligned}
& i_{2}=\rho_{2} \cos \theta_{2} \\
& j_{2}=\rho_{2} \sin \theta_{2}
\end{aligned}
$$

where $\rho_{2}=\mathrm{R}(\rho)$ and $\theta_{2}=\mathrm{T}(\theta)$.

\section{Step3:}

By referencing $\mathrm{R}$ and $\mathrm{T}$ values, original $(\rho, \theta)$ location is recalculated into $\left(\rho_{2}, \theta_{2}\right)$.

Step4:

The recalculated $\left(\rho_{2}, \theta_{2}\right)$ is inverse-transformed into $\left(i_{2}, j_{2}\right)$ pixel location. This is reflected into original image and one can generate result image. Figure 3 shows an example of the proposed method.

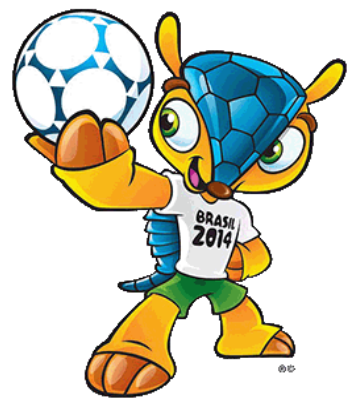

(a)

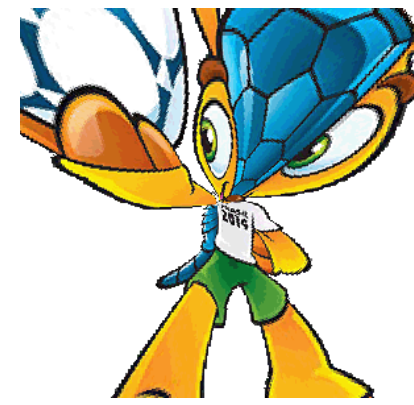

(b)

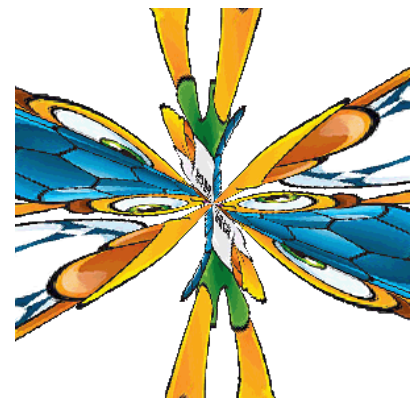

(c) 


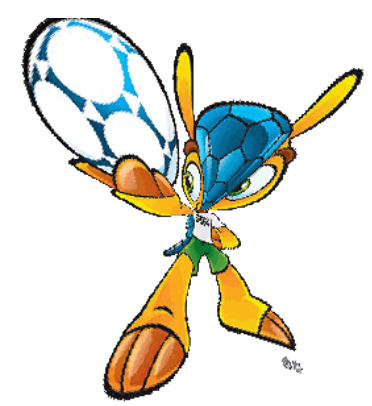

(d)

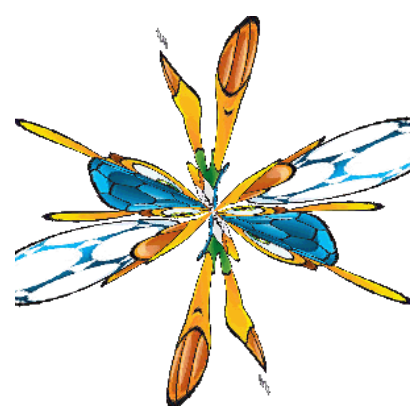

(e)

Figure 3. Examples: (a) Original Image, (b) Result Image with $R=80, T=1$, (c) Result Image with $R=80, T=2$, (d) Result Image with $R=160, T=1$, and (e) Result Image with $R=160, T=2$

\section{Simulation Results}

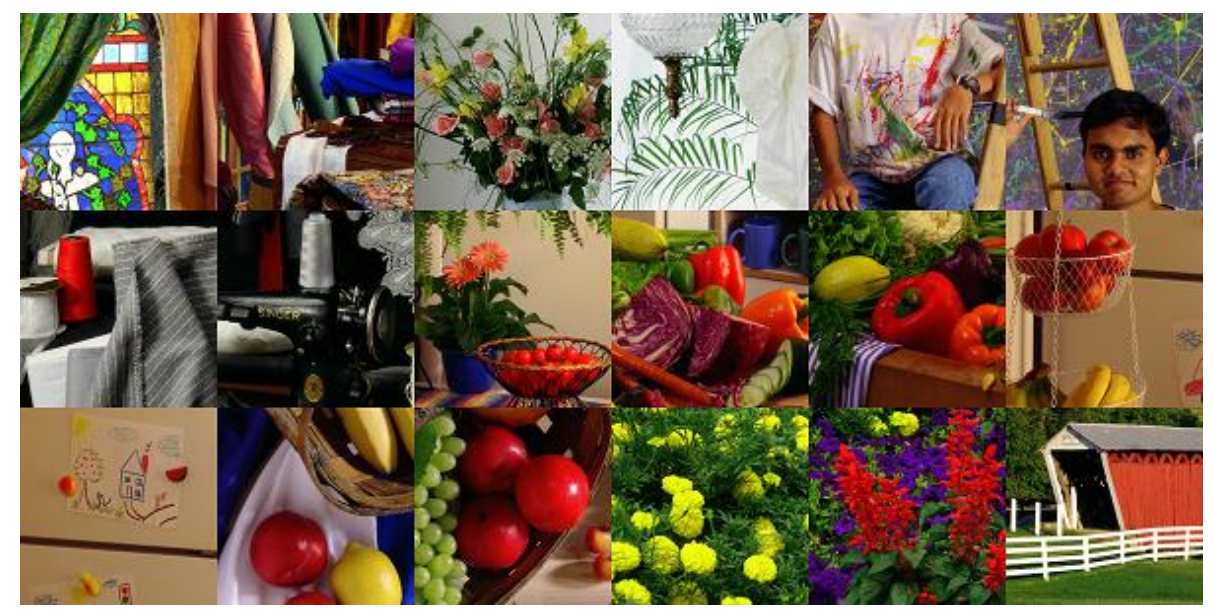

Figure 4. Used Dataset: 18 McM Images

The proposed methodology is applied and tested on exisitg imgaes. The used imagset is McM imageset, which are shown in Figure 4. Objective and subject perofrmance comparsions are provided in this section. The objective mtric is Mean squared error (MSE) metric, which is applied on red channel, green channel, blue channel, and color image. The equation of MSE is determined as,

$$
M S E=\frac{1}{N} \sum_{n=1}^{N}\left(I_{n}-\hat{I}_{n}\right)^{2}
$$

where $I_{n}$ is $n^{\text {th }}$ pixel's intensity. The hat of $I_{n}$ is estimated (or reconstructed) intensity values. Value $N$ is the number of pixels in an image.

Figure 5 shows visual performance comparison results with parameters $(R, T)=(50,1)$, $(50,2),(50,3),(50,4),(50,5)$, and $(50,6)$. As we can see, as parameter $\mathrm{T}$ increases result images are shown $\mathrm{T}$ times on different angles. 


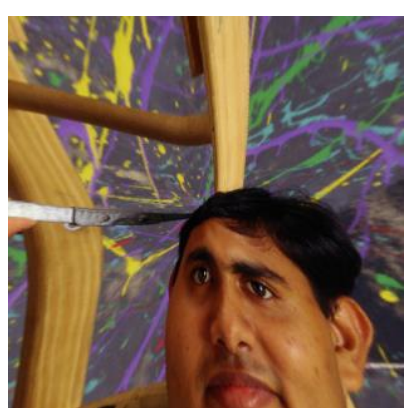

(a)

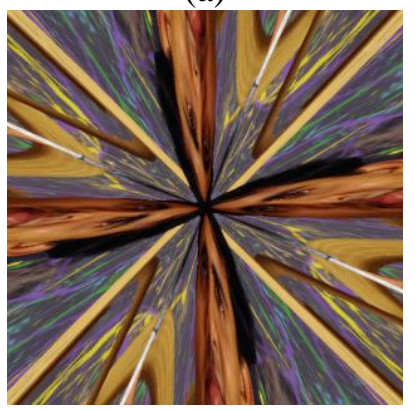

(d)

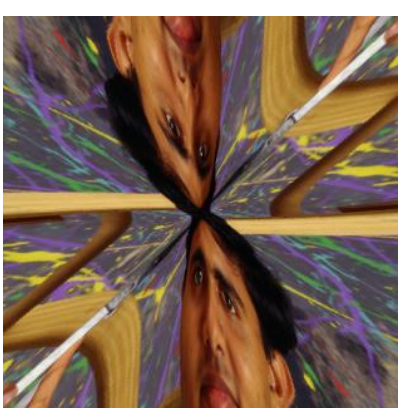

(b)

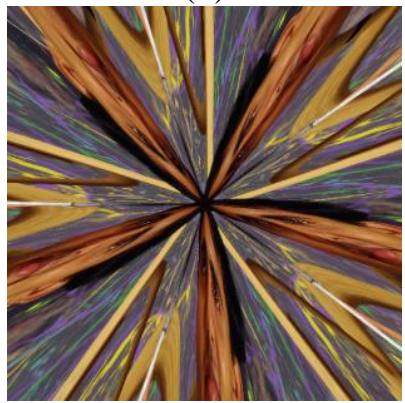

(e)

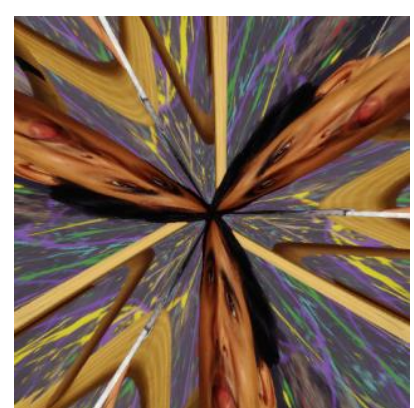

(c)

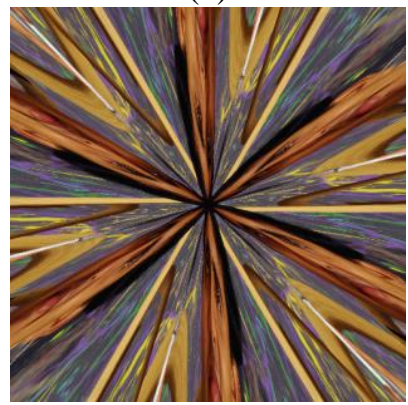

(f)

Figure 5. Simulation Results with $R=50$ on \#6 Image: (a) $T=1$, (b) $T=2$, (c) $\mathrm{T}=3$, (d) $\mathrm{T}=4$, (e) $\mathrm{T}=5$, and (f) $\mathrm{T}=6$

Figure 6 shows the results when $(\mathrm{R}, \mathrm{T})=(100,1),(100,2),(100,3)$, and $(100,4)$ on \#9 McM image.

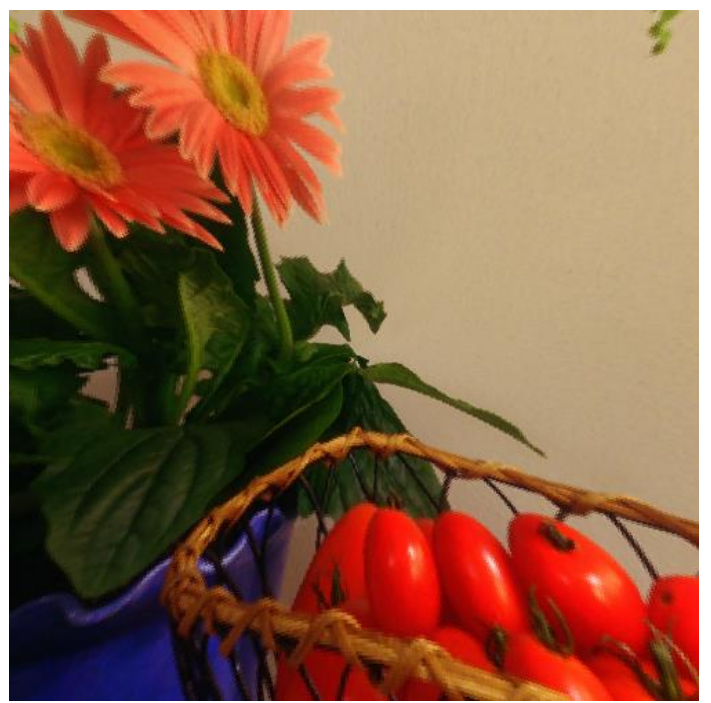

(a)

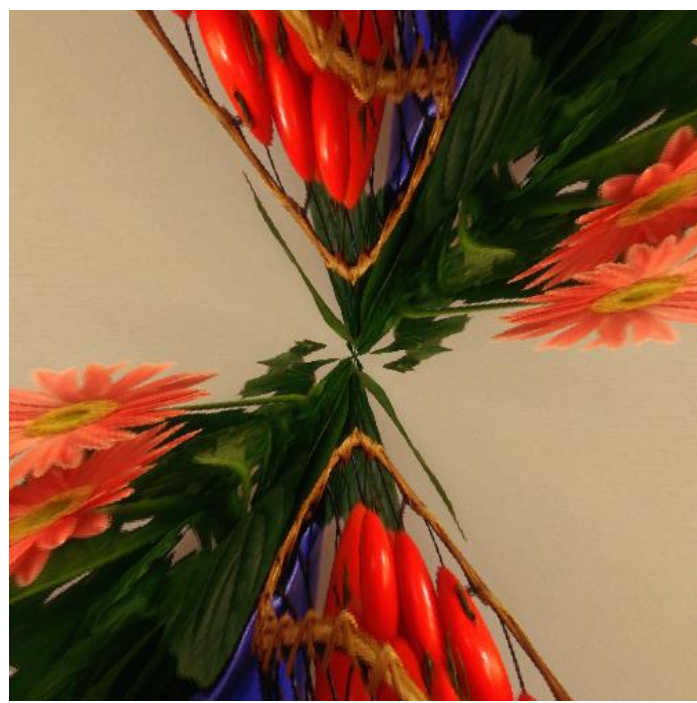

(b) 


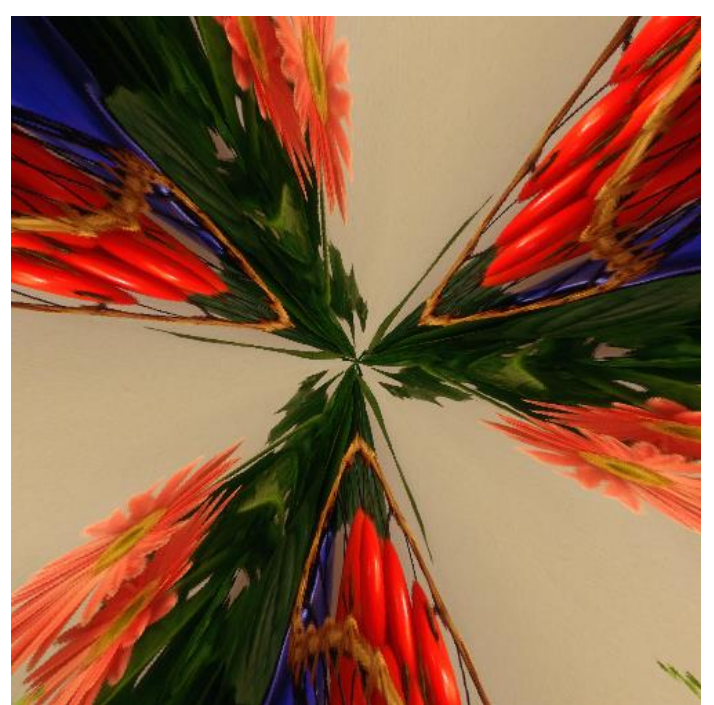

(c)

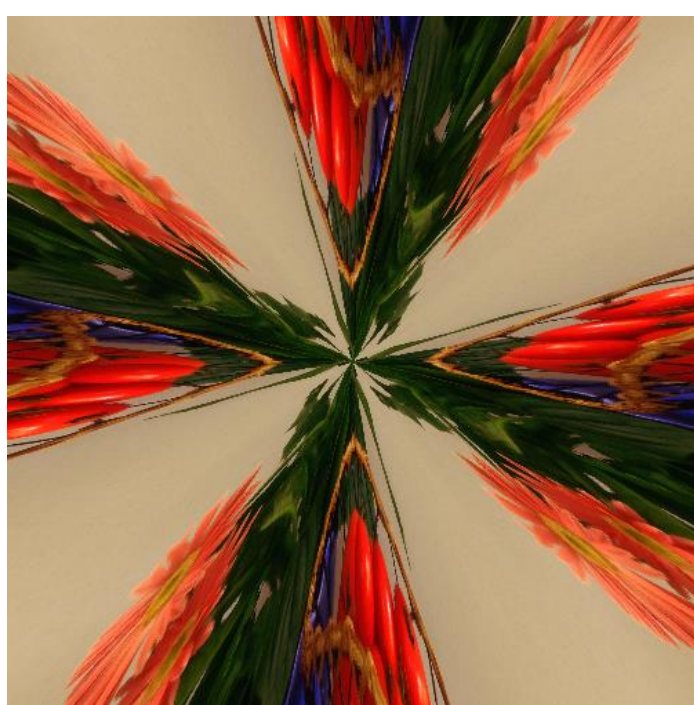

(d)

Figure 6. Simulation Results with $R=100$ on McM \#9 Image: (a) $T=1$, (b) $T=2$, (c) $\mathrm{T}=3$, and (d) $\mathrm{T}=4$

Objective performance comparisons are displayed in Figure 7. The $x$-axis implies image number of McM dataset and the $y$-axis implies MSE results on red channel, green channel, blue channel, and color image. Note that parameter $\mathrm{R}=100$ for this simulation.

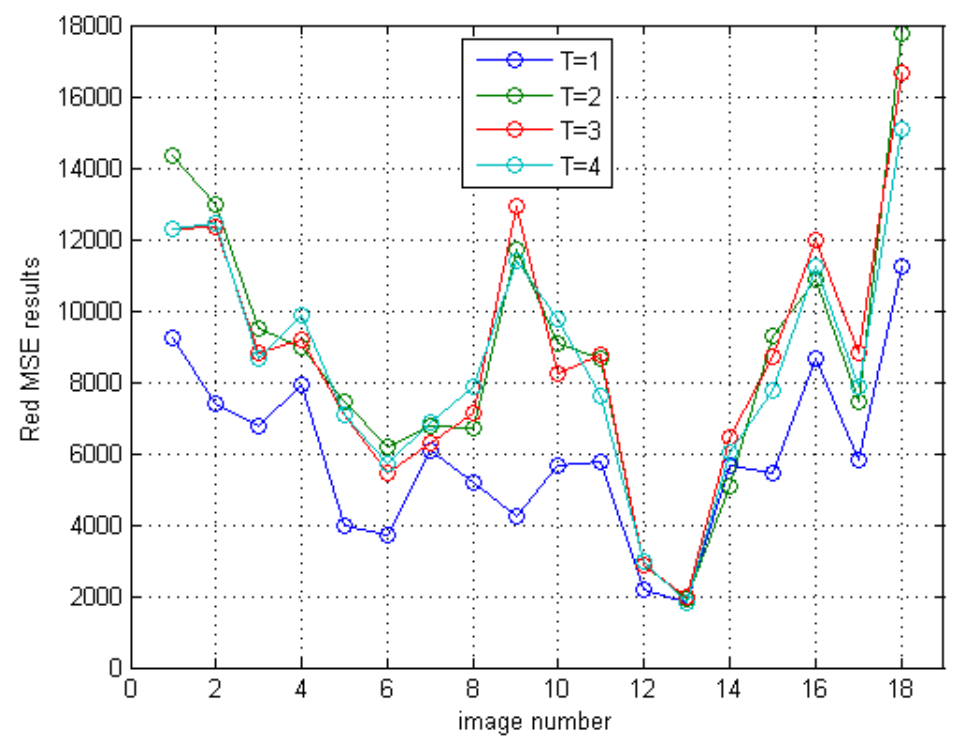

(a) 


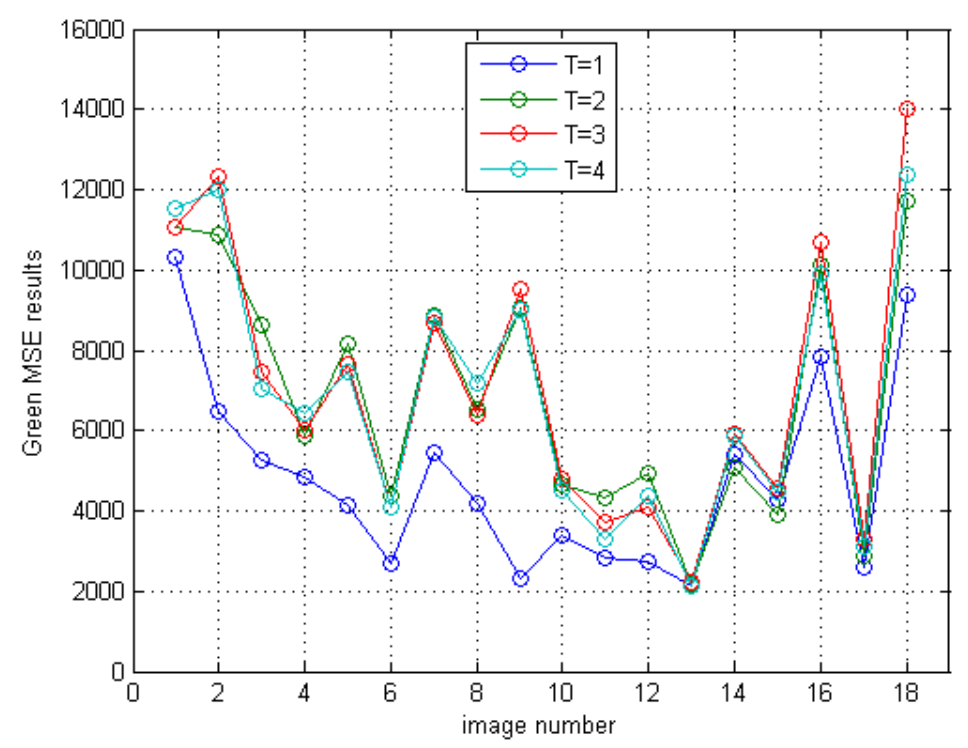

(b)

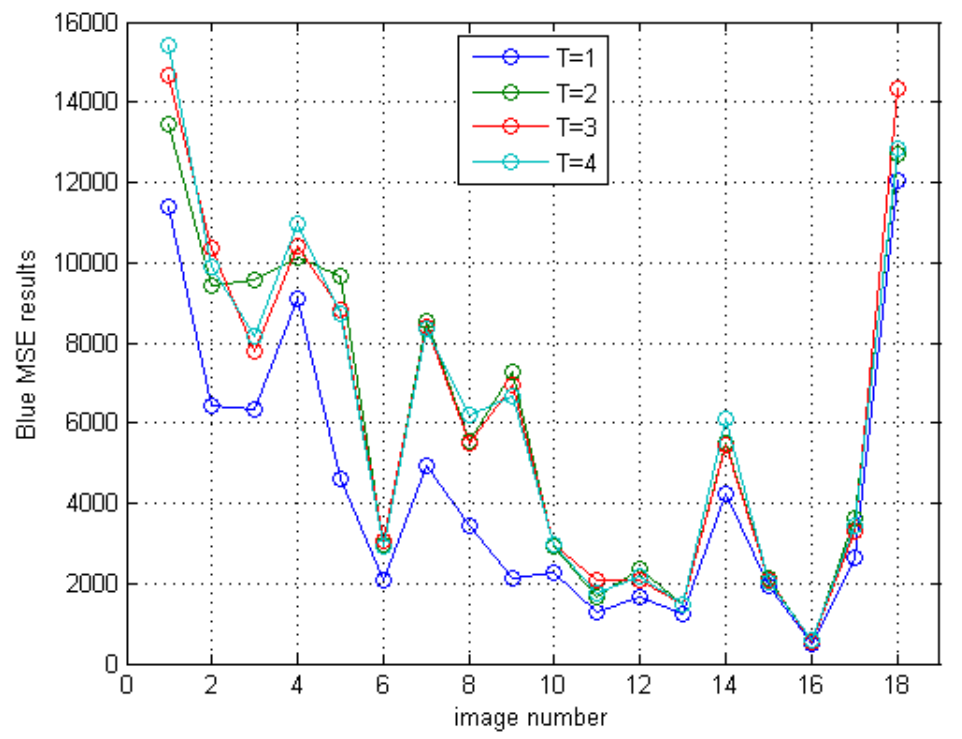

(c) 


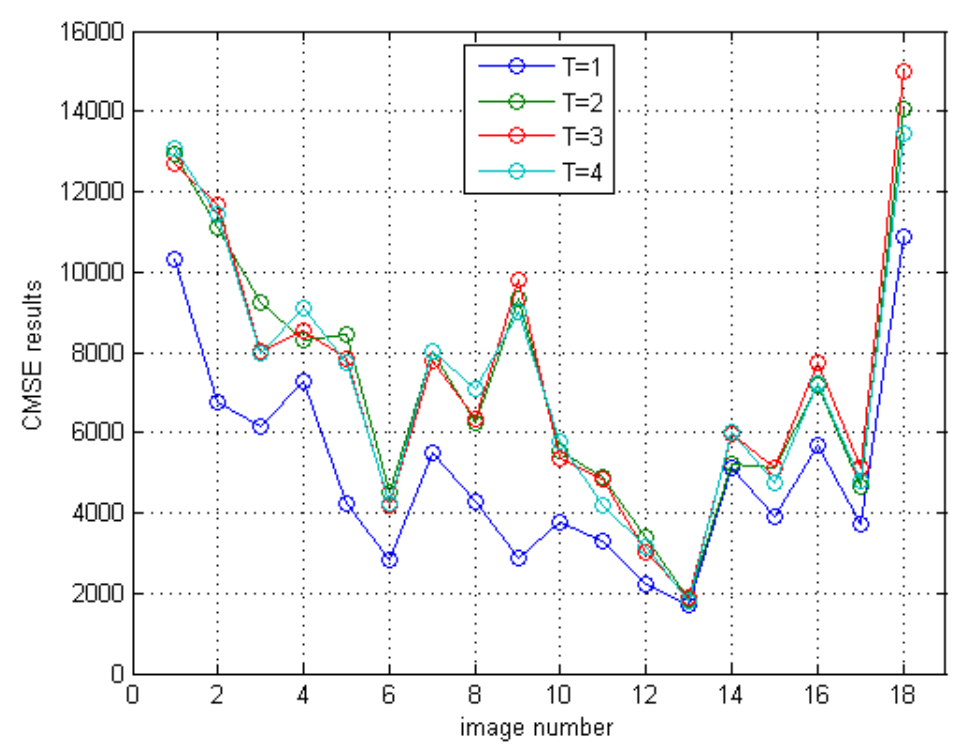

(d)

Figure 7. MSE Performance Comparison with R=100: (a) Red, (b) Green, (c) Blue, and (d) CMSE

Subjective performance comparisons for different $\mathrm{R}$ are provided in Figs. 8 and 9. McM images \#6 and \#18 are used for Figure 8 and 9. Parameter $\mathrm{T}=1$ is given for all cases, and different $\mathrm{R}$ is given for each cases.

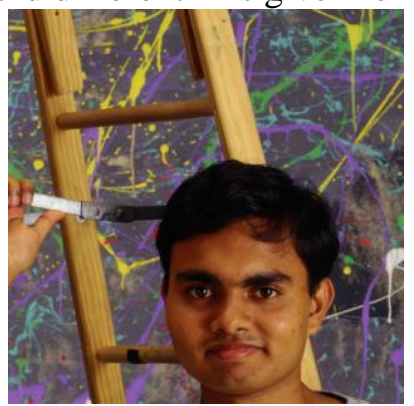

(a)

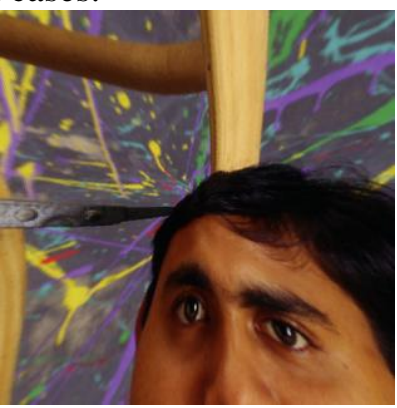

(b)

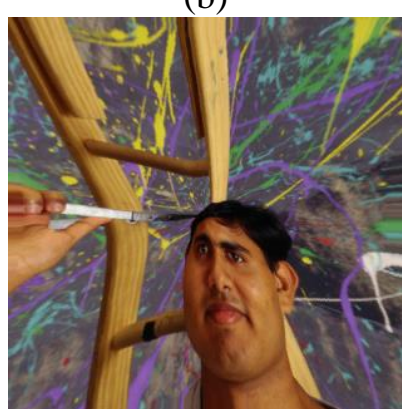

(d)

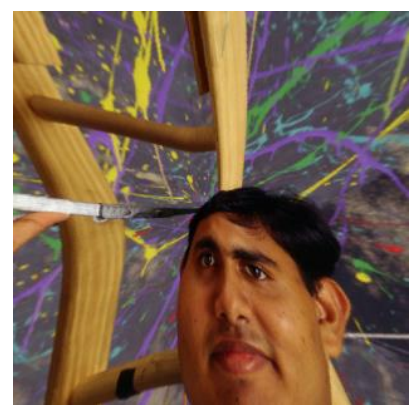

(c)

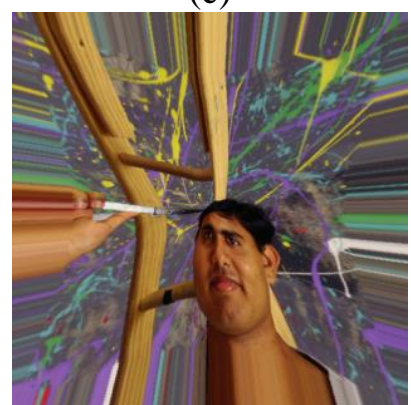

(e)

Figure 8. Simulation Results with $\mathrm{T}=1$ on McM \#6 Image: (a) Original Image, (b) $R=90$, (c) $R=180$, (d) $R=270$, and (e) $R=360$ 


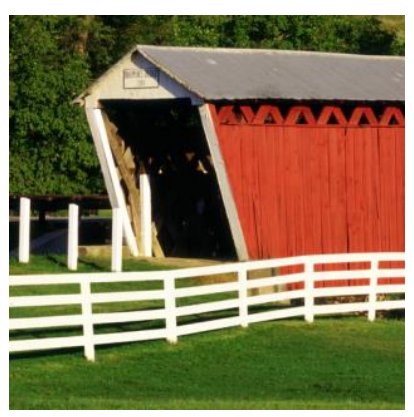

(a)

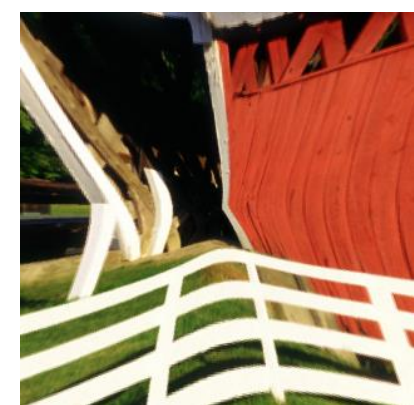

(b)

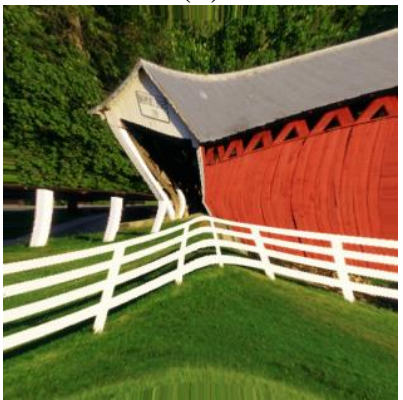

(d)

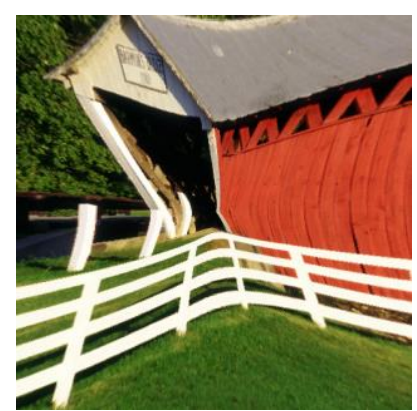

(c)

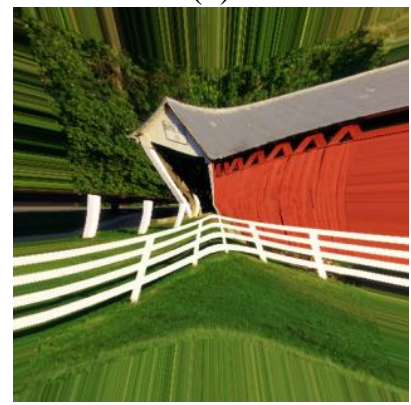

(e)

Figure 9. Simulation Results with $\mathrm{T}=1$ on McM \#18 Images: (a) Original \#18 Image, (b) Result of $R=90$, (c) Result of $R=180$, (d) Result of $R=270$, and (e) Result of $R=360$

The objective performances for different $\mathrm{R}$ are given in Figure 10. Note that given $\mathrm{T}=1$.

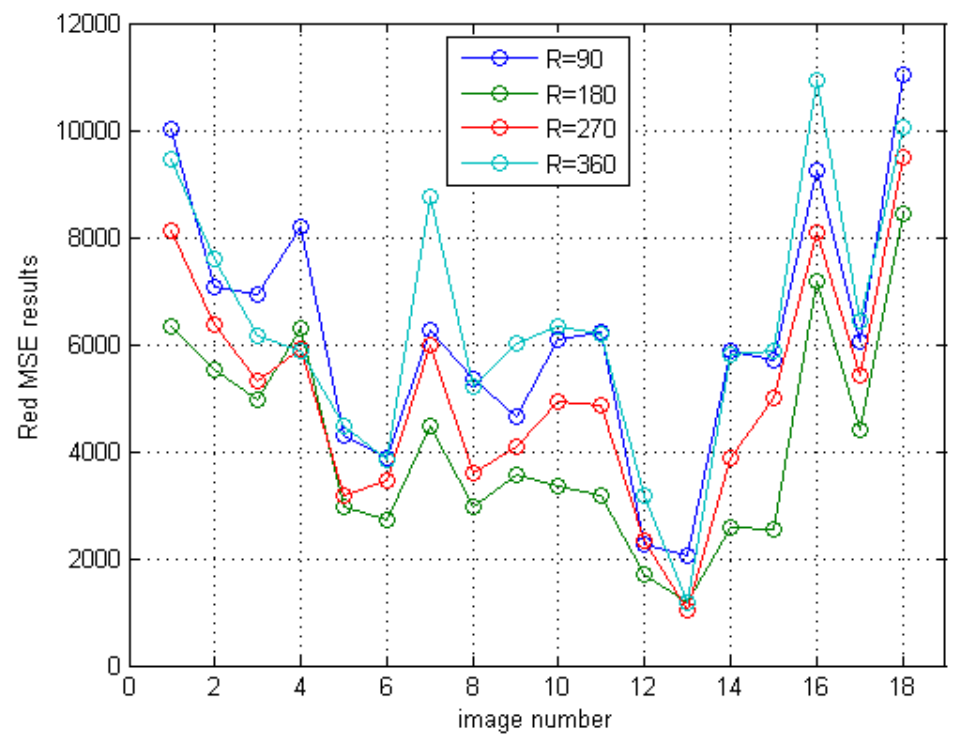

(a) 
International Journal of Multimedia and Ubiquitous Engineering

Vol.10, No.5 (2015)

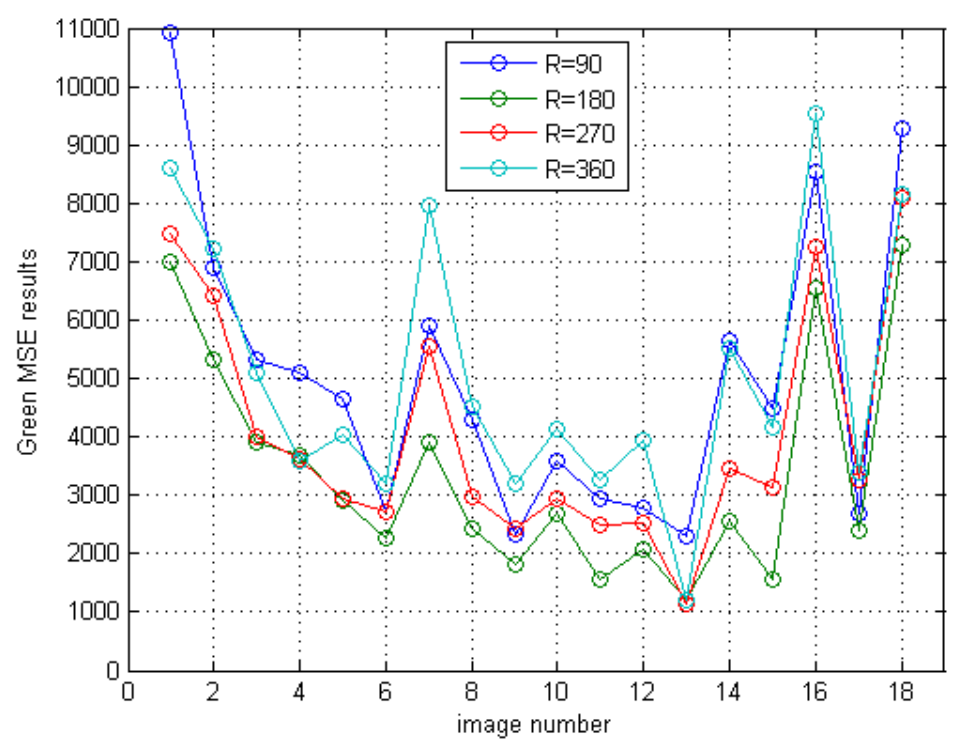

(b)

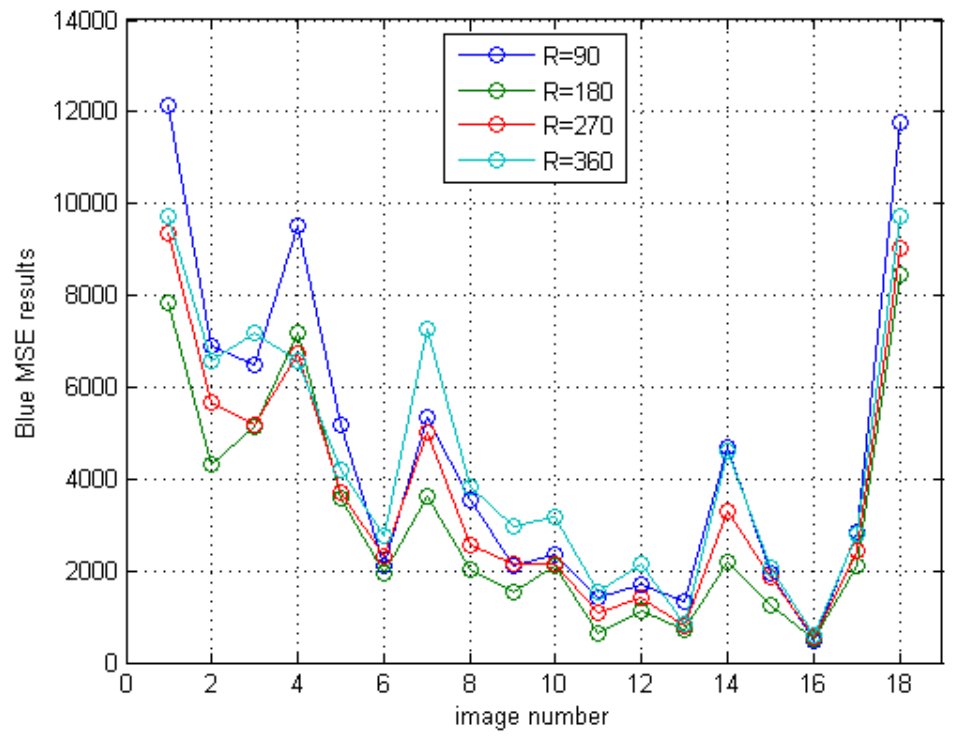

(c) 


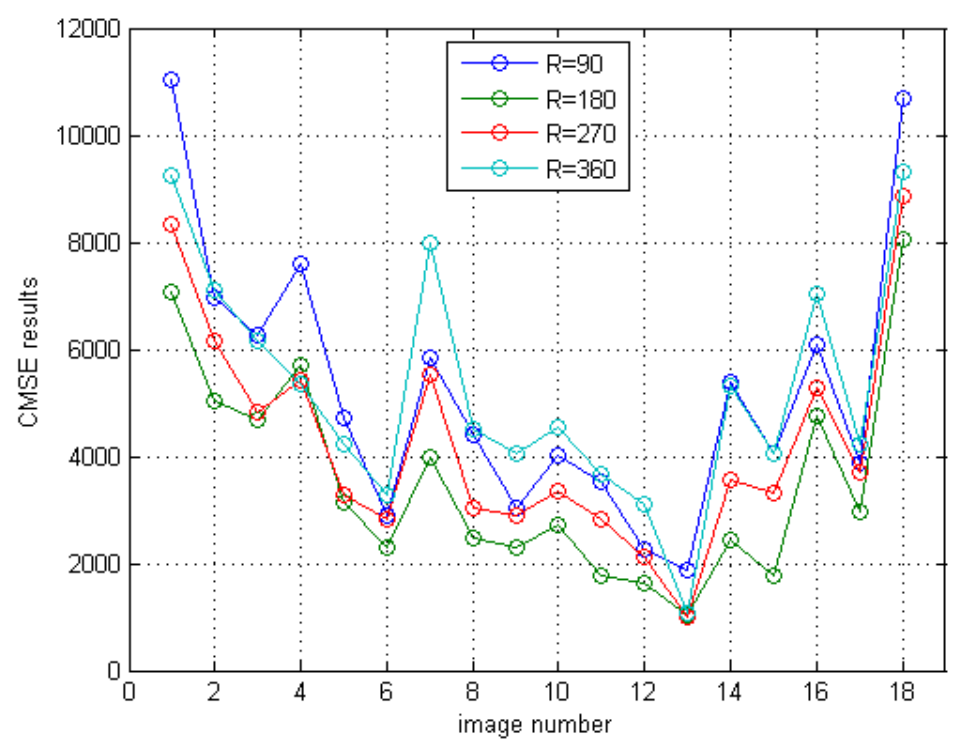

(d)

Figure 10. MSE Performance Comparison with $T=1$ : (a) Red, (b) Green, (c) Blue, and (d) CMSE

\section{Conclusions}

In this paper, we propose a new circular effect generation method. As shown in block diagram of the proposed method, $(i, j)$ coordinate system is changed to $(\rho, \theta)$ where $\left(\rho_{2}, \theta_{2}\right)$ is determined by rules. The inverse transform is applied in $\left(\rho_{2}, \theta_{2}\right)$ to obtain $\left(i_{2}, j_{2}\right)$. Simulation results thoroughly compared objective and subjective performance.

\section{Acknowledgements}

This research was supported by Basic Science Research Program through the National Research Foundation of Korea (NRF) funded by the Ministry of Science, ICT and Future Planning (2014025627)

This paper is a revised and expanded version of a paper entitled "Circular Representation for Images" presented at ASEA2014.

\section{References}

[1] C. Jin, Q.-H. Deng and J. Liu, "Computer Virus Propagation Model Based on Variable Propagation Rate," IJAST, vol. 1, (2008) December, pp. 29-34.

[2] C.-T. Hsieh, Y.-K. Wu, and K.-M. Hung, "Hybrid Watermarking Scheme for Halftone Images," IJAST, vol. 1, (2008) December, pp. 9-20.

[3] S. Lee, J. Yang, and G. Jeon, "Circular Representation for Images," in Proc. ASEA2014, (2014) December.

[4] G. Jeon, M. Anisetti, V. Bellandi, and J. Jeong, "Fuzzy rule-based edge-restoration algorithm in HDTV interlaced sequences," IEEE Trans. Consumer Electronics, vol. 53, no. 2, (2007) May, pp. 725-731.

[5] G. Jeon, M. Anisetti, V. Bellandi, E. Damiani, and J. Jeong, "Rough sets-assisted subfield optimization for alternating current plasma display panel," IEEE Trans. Consumer Electronics, vol. 53, no. 3, (2007) August, pp. 825-832.

[6] G. Jeon, M. Anisetti, V. Bellandi, E. Damiani, and J. Jeong, "Fuzzy weighted approach to improve visual quality of edge-based filtering," IEEE Trans. Consumer Electronics, vol. 53, no. 4, (2007) November, pp. 1661-1667.

[7] G. Jeon, M. Anisetti, J. Lee, V. Bellandi, E. Damiani, and J. Jeong, "Concept of linguistic variable-based fuzzy ensemble approach: application to interlaced HDTV sequences," IEEE Trans. Fuzzy Systems, vol. 17, no. 6, (2009) December, pp. 1245-1258. 
[8] G. Jeon, M.Y. Jung, M. Anisetti, V. Bellandi, E. Damiani, and J. Jeong, "Specification of the geometric regularity model for fuzzy if-then rulebased deinterlacing," IEEE/OSA Journal of Display Technology, vol. 6, no. 6, (2010) June, pp. 235-243.

\section{Author}

Gwanggil Jeon received the $\mathrm{BS}$, MS, and $\mathrm{PhD}$ (summa cum laude) degrees in Department of Electronics and Computer Engineering from Hanyang University, Seoul, Korea, in 2003, 2005, and 2008, respectively.

From 2008 to 2009, he was with the Department of Electronics and Computer Engineering, Hanyang University, from 2009 to 2011, he was with the School of Information Technology and Engineering (SITE), University of Ottawa, as a postdoctoral fellow, and from 2011 to 2012, he was with the Graduate School of Science \& Technology, Niigata University, as an assistant professor. He is currently an assistant professor with the Department of Embedded Systems Engineering, Incheon National University, Incheon, Korea. His research interests fall under the umbrella of image processing, particularly image compression, motion estimation, demosaicking, and image enhancement as well as computational intelligence such as fuzzy and rough sets theories.

He was the recipient of the IEEE Chester Sall Award in 2007 and the 2008 ETRI Journal Paper Award. 